\title{
Legacy Drug-Prescribing Patterns in Primary Care
}

Dee Mangin, $M B C b B, D P H$, FRNZCGP ${ }^{1,2}$

Jennifer Lawson, MLIS ${ }^{1}$

Jessica Cuppage, $M D^{3}$

Elizabeth Shaw, MD, CCFP, CFPC, FCFP'

Katalin Ivanyi, MD, CCFP, FCFP $P^{1,4}$

Amie Davis, MD, CCFP ${ }^{1,5}$

Catby Risdon, MD, DMan, CCFP, FCFP'

'Department of Family Medicine, McMaster University, Hamilton, Ontario, Canada

${ }^{2}$ University of Otago, Christchurch, New Zealand

${ }^{3}$ University of Toronto, Ontario, Canada

${ }^{4}$ Stonechurch Family Health Centre, Hamilton, Ontario, Canada

${ }^{5}$ Halton McMaster Family Health Centre, Burlington, Ontario, Canada

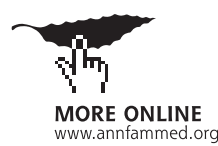

Conflicts of interest: authors report none.

\section{CORRESPONDING AUTHOR}

Dee Mangin, MBChB, DPH, FRNZCGP David Braley Health Sciences Centre McMaster University 100 Main St W, 5th Fl Hamilton, Ontario, Canada L8P 1H6 mangind@mcmaster.ca

\begin{abstract}
PURPOSE Polypharmacy is a key clinical challenge for primary care. Drugs that should be prescribed for an intermediate term (longer than 3 months, but not indefinitely) that are not appropriately discontinued could contribute to polypharmacy. We named this type of prescribing legacy prescribing. Commonly prescribed drugs with legacy prescribing potential include antidepressants, bisphosphonates, and proton pump inhibitors (PPIs). We evaluated the proportion of legacy prescribing within these drug classes.
\end{abstract}

METHODS We conducted a population-based retrospective cohort study using prospectively collected data from the McMaster University Sentinel and Information Collaboration (MUSIC) Primary Care Practice Based Research Network, located in Hamilton, Ontario. All adult patients (aged 18 or older) in the MUSIC data set during 2010-2016 were included $(N=50,813)$. We calculated rates of legacy prescribing of antidepressants (prescription longer than 15 months), bisphosphonates (longer than 5.5 years), and PPIs (longer than 15 months).

RESULTS The proportion of patients having a legacy prescription at some time during the study period was $46 \%(3,766$ of 8,119$)$ for antidepressants, $14 \%(228$ of 1,592$)$ for bisphosphonates, and $45 \%(2,885$ of 6,414$)$ for PPIs. Many of these patients held current prescriptions. The mean duration of prescribing for all legacy prescriptions was significantly longer than that for non-legacy prescriptions $(P<.001)$. Concurrent legacy prescriptions for both antidepressants and PPIs was common, signaling a potential prescribing cascade.

CONCLUSIONS The phenomenon of legacy prescribing appears prevalent. These data demonstrate the potential of legacy prescribing to contribute to unnecessary polypharmacy, providing an opportunity for system-level intervention in primary care with enormous potential benefit for patients.

Ann Fam Med 2018;16:515-520. https://doi.org/10.1370/afm.2315.

\section{INTRODUCTION}

I nappropriate polypharmacy is a key clinical challenge for primary care and has been well described. ${ }^{1-3}$ Concerns about polypharmacy relate to its potential effects on quality of life due to adverse drug reactions, including both direct drug effects (eg, falls, impaired cognition, and poorer nutrition) and drug interactions. Polypharmacy is also associated with reduced medication adherence and difficulties in managing complicated medication regimens that exceed patients' ability to cope. .-9 $^{4-9}$

In Canada, adverse drug reactions cause an estimated 70,000 preventable hospital admissions per year. ${ }^{4}$ Adverse drug reactions requiring medical care affect a substantial proportion of older adults (occurring in 13\% of those on 5 or more medications); one-third are considered preventable. ${ }^{10,11}$

Drivers of polypharmacy, and current strategies to reduce inappropriate medication use and polypharmacy, along with evidence for their effect, are reviewed elsewhere. ${ }^{3}$ Single-disease guidelines have been flagged as unsuitable for use in patients with multimorbidity and just one driver of polypharmacy ${ }^{3,12,13}$; however, other systemic aspects of care and prescribing that contribute to inappropriate polypharmacy are less clear.

Inappropriate prescribing is often conceptualized in drug-based terms: the total numbers, types, or combinations of drugs concurrently pre- 
scribed, for example, as outlined by Beers Criteria or anticholinergic burden. ${ }^{14-16}$ It can also be conceptualized in terms of prescribing duration. Intermediateterm prescribing may be thought of as prescribing that is indicated for more than 3 months but usually not indefinitely. Inappropriate prescribing can occur when, despite initial appropriateness, these drugs are not discontinued after their usual effective or recommended period. We have termed this legacy prescribing and hypothesize that it may represent a substantial issue, contributing to inappropriate polypharmacy.

Primary care serves a coordinating function for patients with multimorbidity and is also the setting for most long-term prescribing, making this an appropriate setting to study and address inappropriate medicine use and polypharmacy. We investigated the extent of legacy drug prescribing for 3 exemplar drug classes prescribed for different conditions-antidepressants, bisphosphonates, and proton pump inhibitors (PPIs) using routinely collected electronic health record prescribing data within a primary care practice-based research network (PBRN).

\section{METHODS}

\section{Study Design and Setting}

We undertook a retrospective cohort study using prospectively collected electronic health record data from January 2010 through December 2016. The study was conducted within the McMaster University Sentinel and Information Collaboration (MUSIC) PBRN in Hamilton, Ontario. Patients served by the MUSIC network represent a wide range of socioeconomic statuses, from distributed neighborhoods within Hamilton and the surrounding area. Practitioners were $60 \%$ female, and the mean year of medical school graduation was 1994 .

\section{Data Source}

The MUSIC data set comprises aggregate, deidentified electronic health record data extracted quarterly and contributed to the Canadian Primary Care Sentinel Surveillance Network (CPCSSN) data set, which is used to describe the epidemiology of primary care in Canada (REB Project Number 14-731). Each new MUSIC data extract is checked and is compared with previous extracts to ensure integrity and stability. From this database, we extracted medications and patient demographics for patients aged 18 to 100 years as of December 31, 2016, as the data set for analysis. Anatomic Therapeutic Chemical (ATC) codes were used to identify specific prescribing data for the 3 drug classes of interest: antidepressants, bisphosphonates, and PPIs (Supplemental Table 1, available at http:// www.annfammed.org/content/16/6/515/suppl/DC1/).

\section{Legacy Prescribing Case Definition}

Legacy prescriptions were identified by calculating prescription durations for each drug class. Our definitions for legacy status for each class were conservative to ensure that this status represented considerable inappropriate exposure. We selected evidence-based inclusion criteria, as follows.

For antidepressants, we used a continuous prescribing duration of longer than 15 months. Treatment is recommended for 6 months after resolution of an acute mood episode and is then stopped in most cases. ${ }^{17}$ Stopping before 6 months results in a higher relapse rate. ${ }^{18}$

For bisphosphonates, we used a continuous prescribing duration of longer than 5.5 years. Treatment for osteoporosis is recommended for up to 5 years in most cases, as the beneficial effect persists beyond 5 years if the medication is stopped at this point. 19,20

For PPIs, we used a continuous prescribing duration of longer than 15 months. Evidence supports only shortterm use (for less than 1 year) of PPIs in most cases. ${ }^{21}$

\section{Data Integrity and Legacy Prescribing Calculation}

The MUSIC database contains a complete set of all prescriptions written by PBRN practices for their enrolled patients and contains data on prescription product, dose, duration, and date. We removed any nonprescribing data appearing as a prescription (for example, notes to the pharmacist), as well as individual prescriptions with erroneous duration values of more than 2,000 days or those with end dates after 2018. All remaining individual prescriptions for each drug class were grouped per patient.

Few studies have described methods for extraction and analysis of medication data from primary care electronic health records, in particular for prescribing duration analysis. ${ }^{22-26}$ In the absence of a standardized approach, we pragmatically developed and tested 2 methods for assessing prescribing duration. Prescription duration using these 2 methods was derived for each patient with prescriptions for the drug classes of interest. One measure, sum duration, was calculated by summing the difference (in days) between the start and stop dates of each prescription, grouped per patient, per drug class. The other measure, start-stop duration, was derived from the difference between the first-ever start date and the last-ever stop date for each drug class grouping per patient.

\section{Legacy Prescribing Case Validation}

To determine the most accurate duration measure, we applied the legacy criteria to patients' sum duration and start-stop duration value pairs, and coded patients to 1 of 4 categories per drug class of interest: (1) legacy based on sum duration only; $(2)$ legacy based on start- 
stop duration only; (3) legacy based on both sum duration and start-stop duration; or (4) nonlegacy based on absence of both duration criteria. Next, the raw prescribing data from a random selection of patients from each of the 4 categories were examined to confirm or refute the legacy assignment and gauge the accuracy of each duration method.

For start-stop duration, we checked for any considerable time gaps in continuous prescribing series of 6 months or longer for PPIs and antidepressants and 1 year or longer for bisphosphonates. Where we detected discontinuous prescribing, we calculated new durations for continuous prescriptions. For values no longer satisfying legacy criteria, we noted the patient's legacy group assignment as inaccurate (these prescriptions may, for example, represent appropriate intermittent prescribing for recurrence or relapse).

We examined the sum duration data to detect any anomalies in the recording of prescriptions. We found duplicate prescriptions, overlapping prescriptions, and prescriptions of 0 days in duration that were contributing to inaccuracy in sum duration measures.

The data validation process demonstrated that the sum duration method was compromised by certain data-recording inaccuracies and the start-stop duration method was unreliable when intermittent prescribing occurred in a prescribing series. In comparison, the start-stop duration method was reasonably accurate for detecting legacy prescribing in a prescription series, but was more robust when the associated sum duration value also satisfied legacy criteria. This validation step confirmed that the most correct estimate of legacy prescribing for a given drug class is when botb sum duration and start-stop duration criteria are satisfied, and we therefore used this definition in our study. Similarly, only patients who did not meet criteria for both sum duration and start-stop were considered true nonlegacy patients in this study. All patients satisfying only sum legacy criteria or only start-stop legacy criteria were included in the denominator of total patients ever prescribed the drug class, but were left out of all subsequent study analyses comparing characteristics of legacy prescription and nonlegacy prescription patient groups. (Supplemental Table 2, available at http://www.annfammed.org/
content/16/6/515/supp1/DC1/ provides the detailed validation data and statistical analyses.)

We calculated the proportion of current patients who still had active legacy prescriptions at the end of the study period (January 1,2017) among patients with an active status in the electronic health record on December 31, 2016.

Figure 1 illustrates this delineation of legacy and nonlegacy prescription patients for the antidepressant drug class, including subsets defined by active patient status and current drug prescriptions. We applied this same sorting technique to the other 2 drug classes to arrive at their subsets for analyses.

\section{RESULTS}

Analyses were based on 50,813 adult patients. Table 1 outlines the summative counts and proportions of

\section{Figure 1. Patients prescribed antidepressants: inclusion and exclusion for comparative analysis.}

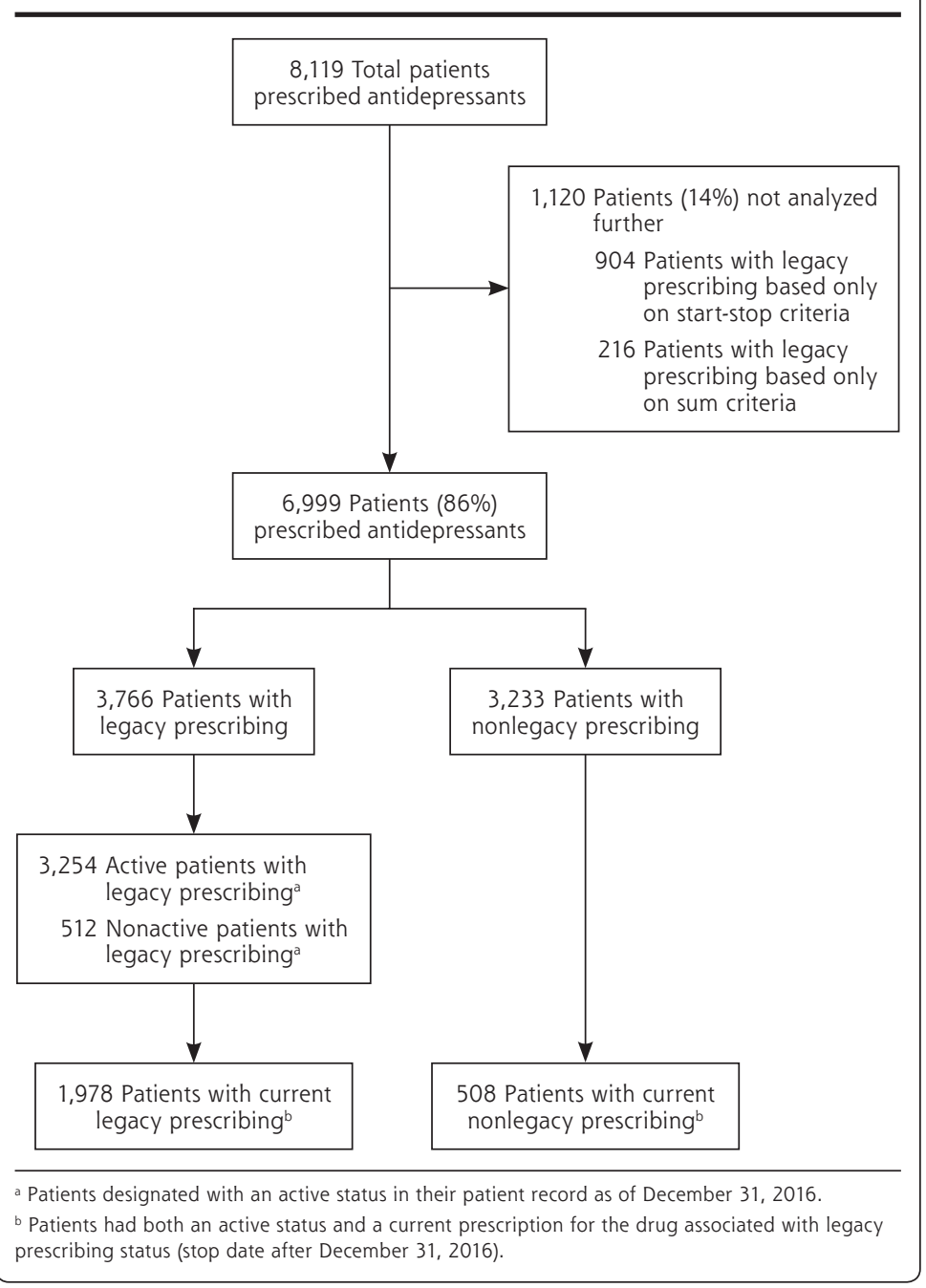


Table 1. Legacy Prescriptions Among Patients Prescribed Each Index Drug Class During 2010-2016

\begin{tabular}{lcccc}
\hline Measure & $\begin{array}{c}\text { Antidepressant } \\
\text { Prescriptions } \\
(\mathbf{n = 8 , 1 1 9 )}\end{array}$ & $\begin{array}{c}\text { Bisphosphonate } \\
\text { Prescriptions } \\
\mathbf{( n = 1 , 5 9 2 )}\end{array}$ & $\begin{array}{c}\text { PPI } \\
\text { Prescriptions } \\
\mathbf{( n = 6 , 4 1 4 )}\end{array}$ & $\begin{array}{c}\text { Total } \\
\text { Prescriptions } \\
\mathbf{( N = 1 6 , 1 2 5 )}\end{array}$ \\
\hline Total included in comparative analysis, No. (\%) & $6,999(86)$ & $1,387(87)$ & $5,660(88)$ & $14,046(87)$ \\
$\quad$ Legacy, No. (\%) & $3,766(46)$ & $228(14)$ & $2,885(45)$ & $6,879(43)$ \\
$\quad$ Nonlegacy, No. (\%) & $3,233(40)$ & $1,159(73)$ & $2,775(43)$ & $7,167(44)$ \\
Mean (SD) prescription duration & & & & - \\
Legacy prescriptions, $y^{\mathrm{a}}$ & $4.8(2.0)^{\mathrm{b}}$ & $6.7(0.6)^{\mathrm{b}}$ & $4.9(1.9)^{\mathrm{b}}$ & - \\
$\quad$ Nonlegacy prescriptions, $y^{\mathrm{c}}$ & $0.4(0.3)$ & $2.0(1.4)$ & $0.4(0.3)$ & - \\
Total excluded from comparative analysis, No. (\%) & $1,120(14)$ & $205(13)$ & $754(12)$ & $2,079(13)$ \\
$\quad$ Only start-stop legacy criteria met, No. (\%) & $904(11)$ & $123(8)$ & $625(10)$ & $1,652(10)$ \\
Only sum legacy criteria met, No. (\%) & $216(3)$ & $82(5)$ & $129(2)$ & $427(3)$ \\
\hline
\end{tabular}

MUSIC = McMaster University Sentinel and Information Collaboration; PPI = proton pump inhibitor.

Note: Data represent counts of individual prescriptions among 50,813 patients (population N) in the MUSIC data set.

a Calculated for patients with an active status and limited to start-stop duration values that were less than 8 years.

Difference between legacy and nonlegacy groups was significant $(P<.001)$.

Calculated for patients with an active status and limited to start-stop duration values that were greater than 0 days.

patients prescribed drugs within each of the 3 drugs classes, and shows whether prescribing was legacy or not. The overall proportion of legacy vs nonlegacy prescribing was fairly evenly split: $43 \%$ and $44 \%$, respectively. The mean proportion of prescribers' patients receiving legacy prescription was $10 \%$ (25th percentile $=7 \%$, 75 th percentile $=12 \%$; interquartile range $=5 \%$ ).

The pooled total number of patients with legacy prescriptions across the 3 drug classes $(6,879)$ amounted to 5,806 unique patients (10\% among all 50,813 patients within the PBRN data set for analysis), indicating that certain patients received legacy prescriptions in more than 1 class. Table 2 shows the patterns of single and dual legacy prescriptions for these patients. Of these unique patients, $17 \%(969$ of 5,806$)$

\section{Table 2. Legacy Prescription and Coprescription: Unique Patients With Single- or Multiple-Drug Legacy Prescriptions, 2010-2016}

\begin{tabular}{lc}
\hline Drug Class/Classes & $\begin{array}{c}\text { Unique Patients, } \\
\text { No. (\%) } \\
\text { (n= 5,806) }\end{array}$ \\
\hline Single-drug legacy prescription & $4,745(82)$ \\
PPI & $1,850(32)$ \\
Antidepressant & $2,759(48)$ \\
Bisphosphonate & $136(2)$ \\
Multiple-drug legacy prescriptions & $1,061(18)$ \\
Antidepressant, bisphosphonate, and PPI & $12(0.2)$ \\
Antidepressant and PPI & $969(17)$ \\
Antidepressant and bisphosphonate & $26(0.4)$ \\
PPI and bisphosphonate & $54(0.9)$ \\
\hline PPI = proton pump inhibitor. & \\
Note: Data represent prescribing patterns among the 5,806 unique patients \\
receiving the 6,879 legacy prescriptions in the second row of Table 1.
\end{tabular}

had legacy prescriptions for both antidepressants and PPIs. No other drug class combination showed notable dual legacy status.

A large proportion of patients who experienced legacy prescribing during the study period still had current active prescriptions for these medications at the end of the study period (61\% of those for antidepressants, $65 \%$ of those for PPIs, $77 \%$ of those for bisphosphonates) (data not shown). A greater proportion of women received legacy prescriptions for antidepressants and bisphosphonates, but not for PPIs, compared with respective men prescribed the same drug classes (Supplemental Table 3, available at http://www.annfammed. org/content/16/6/515/suppl/DC1/). Across all drug classes, patients with legacy prescribing were relatively older than counterparts with nonlegacy prescribing.

\section{DISCUSSION}

\section{Main Study Findings}

Our findings suggest that legacy prescribing is prevalent, is consistent across prescribers, and could be an important system-level contributor to inappropriate polypharmacy. The high proportion of currently active legacy prescriptions found represents an opportunity for both research and improvement in patient care.

The high level of coprescription of antidepressants and PPIs may represent an important and previously unreported prescribing cascade, whereby the adverse effects of an index medication mimic the symptoms of a disorder, for which another medication is then prescribed. ${ }^{27}$ Selective serotonin reuptake inhibitors, which are the most commonly prescribed antidepressant class, have substantial gastrointestinal effects, supporting this potential association. 


\section{Strengths and Limitations}

We used routinely collected data from a PBRN to provide valuable practice-based evidence on longitudinal prescribing patterns in a real-world primary care setting. ${ }^{28}$ With no validated standard methodology previously described in the literature, we pragmatically developed and validated one that reasonably estimates prescribing duration. This method can be applied and refined in other health care settings to define rates of legacy drug prescribing, serving as a useful canary in the coal mine signal of systemic prescribing issues.

There are important limitations linked to the nature of the data. Although many appropriate intermittent prescriptions will have been excluded by requiring both start-stop and sum legacy criteria to be filled, a certain proportion of prescriptions meeting these criteria will be clinically appropriate given specific patient characteristics, and that determination was beyond the scope of this study. For example, some guidelines suggest longer-duration antidepressant therapy (up to 2 years or longer) for certain limited patient subgroups. This recommendation, though weak (opinion-based Strength-ofRecommendation Taxonomy $=$ C) and not supported by evidence of effectiveness in the primary care population, may explain some of the effect seen. ${ }^{17,29-36}$ Nonetheless, the data presented indicate long-term prescribing levels and mean durations far exceed what might be expected.

Of the 3 drugs classes studied, we found comparatively fewer legacy bisphosphonate prescriptions, possibly because the duration of the cohort data available is close to the legacy duration definition for this drug class. Future availability of longitudinal data will clarify this finding.

Finally, the data we used represented prescribing and not dispensing, so they likely overestimate patient exposure. On the other hand, this study did not include other concurrent, specialist-prescribing data outside of the MUSIC PBRN, potentially leading to an underestimate of legacy prescribing.

\section{Study Implications}

Legacy prescribing appears to be an important contributor to inappropriate prescribing. Although noted in theory as a prescribing fault, inappropriate duration has been largely invisible as a source of inappropriate prescribing. This invisibility may occur because studies of error are largely undertaken in secondary care settings and because drug-based assessments are used. ${ }^{37-39}$ Prescribing systems are largely geared toward starting and continuing medicines; most have no controls to flag the end of an intermediate-term prescription, while systems and software features for routine prescription refilling are common. Our results are therefore not surprising and indicate a need for system-oriented change that encompasses prescribing systems, education, and patient-pharmacist-physician communication on appropriate stopping of drug therapy. ${ }^{39,40}$

Labor-intensive audit and feedback solutions are often workarounds for system flaws, and can only discover when potentially inappropriate prescribing has already occurred. We suggest the best timing for interventions will be at initial and any repeated prescription, aiming to preempt legacy prescribing with patients as an essential partner in shared decision making.

There is fertile ground here to improve prescribing and reduce unnecessary overtreatment, polypharmacy, morbidity, and costs associated with adverse drug reactions. ${ }^{12,41}$ Legacy prescribing could also be explored as a quality measure incentivizing restraint in a system where there are few, if any, current indicators of the adverse effects of too much medicine. ${ }^{42}$

To read or post commentaries in response to this article, see it online at http://www.AnnFamMed.org/content/16/6/515.

Key words: polypharmacy; bisphosphonates; antidepressive agents; proton pump inhibitors; family practice; primary health care; health services research; electronic health records; potentially inappropriate medication list; inappropriate prescribing; practice-based research

Submitted March 23, 2018; submitted, revised, August 27, 2018; accepted September 10, 2018.

Funding support: Funding for this research was provided by the Pilot Research Project Fund, Department of Family Medicine, McMaster University.

Previous presentations: Mangin D, Lawson J, Cuppage J, et al. Legacy drug prescribing patterns and associations in primary care: cohort study in the MUSIC. Poster presented at the Trillium Research Day Conference; May 31, 2017 practice based research network; Toronto, Ontario, Canada.

Mangin D, Lawson J, Cuppage J, et al. Legacy drug prescribing patterns and associations in primary care: cohort study in the MUSIC practice based research network. Poster presented at the NAPCRG Annual Conference; Nov 19, 2017; Montreal, Quebec, Canada.

Mangin D, Lawson J, Cuppage J, et al. Legacy drug prescribing patterns and associations in primary care: cohort study in the MUSIC practice based research network. Oral presentation at the NAPCRG Practice-based Research Network Conference; June 26, 2018; Bethesda, Maryland.

Acknowledgments: The authors would like to thank the primary care clinicians and patients of the MUSIC PBRN who contribute their data to the network through which the study data were generated; Krzysztof Adamczyk, the IT lead for the MUSIC network; and the support of the McMaster University Department of Family Medicine for this PBRN.

Supplemental Materials: Available at http://www.AnnFamMed. org/content/16/6/515/suppl/DC1/.

\section{References}

1. McCarthy L, Dolovich L, Haq M, Thabane L, Kaczorowski J. Frequency of risk factors that potentially increase harm from medications in older adults receiving primary care. Can J Clin Pharmacol. 2007;14(3):e283-e290. 
2. Salisbury C, Johnson L, Purdy S, Valderas JM, Montgomery AA. Epidemiology and impact of multimorbidity in primary care: a retrospective cohort study. Br J Gen Pract. 2011;61(582):e12-e21.

3. Mangin D, Bahat G, Golomb BA, et al. International Group for Reducing Inappropriate Medication Use \& Polypharmacy (IGRIMUP): Position statement and 10 recommendations for action. Drugs Aging. 2018;35(7):7575-7587.

4. Baker GR, Norton PG, Flintoft V, et al. The Canadian Adverse Events Study: the incidence of adverse events among hospital patients in Canada. CMAJ. 2004;170(11):1678-1686.

5. Carleton B, Smith M. Drug safety: side effects and mistakes or adverse reactions and deadly errors? BCMJ. 2006;48(7):329-333.

6. Frazier SC. Health outcomes and polypharmacy in elderly individuals: an integrated literature review. J Gerontol Nurs. 2005;31(9):4-11.

7. Hilmer SN, Gnjidic D. The effects of polypharmacy in older adults. Clin Pharmacol Ther. 2009;85(1):86-88.

8. May C, Montori VM, Mair FS. We need minimally disruptive medicine. BMJ. 2009;339(Aug 11):b2803.

9. Tinetti ME, Bogardus ST Jr, Agostini JV. Potential pitfalls of diseasespecific guidelines for patients with multiple conditions. $N$ Engl J Med. 2004;351(27):2870-2874.

10. Canadian Institute for Health Information. Seniors and the health care system: what is the impact of multiple chronic conditions? https://secure.cihi.ca/free_products/air-chronic_disease_aib_en.pdf. Published Jan 2011. Accessed Jan 17, 2018.

11. George CJ, Korc B, Ross JS. Appropriate proton pump inhibitor use among older adults: a retrospective chart review. Am J Geriatr Pharmacother. 2008;6(5):249-254.

12. Boyd CM, Darer J, Boult C, Fried LP, Boult L, Wu AW. Clinical practice guidelines and quality of care for older patients with multiple comorbid diseases: implications for pay for performance. JAMA. 2005;294(6):716-724.

13. Mangin $D$, Heath I, Jamoulle M. Beyond diagnosis: rising to the multimorbidity challenge. BMJ. 2012;344:e3526.

14. Wallace J, Paauw DS. Appropriate prescribing and important drug interactions in older adults. Med Clin North Am. 2015;99(2):295-310.

15. Salahudeen MS, Duffull SB, Nishtala PS. Anticholinergic burden quantified by anticholinergic risk scales and adverse outcomes in older people: a systematic review. BMC Geriatr. 2015;15:31.

16. American Geriatrics Society 2015 Beers Criteria Update Expert Panel. American Geriatrics Society 2015 updated Beers criteria for potentially inappropriate medication use in older adults. J Am Geriatr Soc. 2015;63(11):2227-2246.

17. Kennedy SH, Lam RW, McIntyre RS, et al; CANMAT Depression Work Group. Canadian Network for Mood and Anxiety Treatments (CANMAT) 2016 clinical guidelines for the management of adults with major depressive disorder: Section 3. Pharmacological treatments. Can J Psychiatry. 2016;61(9):540-560.

18. Borges $S$, Chen YF, Laughren TP, et al. Review of maintenance trials for major depressive disorder: a 25-year perspective from the US Food and Drug Administration. J Clin Psychiatry. 2014;75(3): 205-214.

19. Black DM, Schwartz AV, Ensrud KE, et al; FLEX Research Group. Effects of continuing or stopping alendronate after 5 years of treatment: the Fracture Intervention Trial Long-term Extension (FLEX): a randomized trial. JAMA. 2006;296(24):2927-2938.

20. Black DM, Reid IR, Boonen S, et al. The effect of 3 versus 6 years of zoledronic acid treatment of osteoporosis: a randomized extension to the HORIZON-Pivotal Fracture Trial (PFT). J Bone Miner Res. 2012;27(2):243-254.

21. Boghossian TA, Rashid FJ, Thompson W, et al. Deprescribing versus continuation of chronic proton pump inhibitor use in adults. Cochrane Database Syst Rev. 2017:3:CD011969.
22. Burt J, Elmore N, Campbell SM, Rodgers S, Avery AJ, Payne RA. Developing a measure of polypharmacy appropriateness in primary care: systematic review and expert consensus study. BMC Med. 2018;16(1):91.

23. Deyo RA, Smith DH, Johnson ES, et al. Opioids for back pain patients: primary care prescribing patterns and use of services. J Am Board Fam Med. 2011;24(6):717-727.

24. Hazra NC, Dregan A, Jackson S, Gulliford MCD. Drug utilization and inappropriate prescribing in centenarians. J Am Geriatr Soc. 2016;64(5):1079-1084.

25. Josephson CB, Gonzalez-Izquierdo A, Denaxas S, et al. Serotonin reuptake inhibitors and mortality in epilepsy: a linked primary-care cohort study. Epilepsia. 2017;58(11):2002-2009.

26. Nauta KJ, Groenhof F, Schuling J, et al. Application of the STOPP/ START criteria to a medical record database. Pharmacoepidemiol Drug Saf. 2017;26(10):1242-1247.

27. Rochon PA, Gurwitz JH. The prescribing cascade revisited. Lancet. 2017;389(10081):1778-1780.

28. Green LW. Making research relevant: if it is an evidence-based practice, where's the practice-based evidence? Fam Pract. 2008 25(Suppl 1):i20-i24.

29. Burcusa SL, Iacono WG. Risk for recurrence in depression. Clin Psychol Rev. 2007;27(8):959-985.

30. Geddes JR, Carney SM, Davies C, et al. Relapse prevention with antidepressant drug treatment in depressive disorders: a systematic review. Lancet. 2003;361(9358):653-661.

31. Piek E, van der Meer K, Nolen WA. Guideline recommendations for long-term treatment of depression with antidepressants in primary care-a critical review. Eur J Gen Pract. 2010;16(2):106-112.

32. Alonso J, Angermeyer MC, Bernert S, et al; ESEMeD/MHEDEA 2000 Investigators, European Study of the Epidemiology of Mental Disorders (ESEMeD) Project. Use of mental health services in Europe: results from the European Study of the Epidemiology of Mental Disorders (ESEMeD) project. Acta Psychiatr Scand Suppl. 2004;(420):47-54.

33. Andrews G, Henderson S, Hall W. Prevalence, comorbidity, disability and service utilisation. Overview of the Australian National Mental Health Survey. Br J Psychiatry. 2001;178(2):145-153.

34. Hickie IB. Primary care psychiatry is not specialist psychiatry in general practice. Med J Aust. 1999;170(4):171-173.

35. van Weel-Baumgarten EM, Schers HJ, van den Bosch WJ, van den Hoogen HJ, Zitman FG. Long-term follow-up of depression among patients in the community and in family practice settings: a systematic review. J Fam Pract. 2000;49(12):1113-1120.

36. Mangin D, Dowson C, Mulder $R$, et al. The effectiveness of maintenance SSRI treatment in primary care depression to prevent recurrence: multicentre double blinded placebo controlled RCT. Paper presented at: 43rd North American Primary Care Research Group Annual Meeting; October 24-28, 2015; Cancun, Mexico.

37. Koper D, Kamenski G, Flamm M, Böhmdorfer B, Sönnichsen A. Frequency of medication errors in primary care patients with polypharmacy. Fam Pract. 2013;30(3):313-319.

38. Sheikh A, Panesar SS, Larizgoitia I, Bates DW, Donaldson LJ. Safer primary care for all: a global imperative. Lancet Glob Health. 2013; 1(4):e182-e183.

39. Velo GP, Minuz P. Medication errors: prescribing faults and prescription errors. Br J Clin Pharmacol. 2009;67(6):624-628.

40. Nolan TW. System changes to improve patient safety. BMJ. 2000; 320(7237):771-773.

41. Levinson W, Kallewaard M, Bhatia RS, Wolfson D, Shortt S, Kerr EA; Choosing Wisely International Working Group. 'Choosing Wisely': a growing international campaign. BMJ Qual Saf. 2015;24(2):167-174.

42. Caverly TJ, Combs BP, Moriates C, Shah N, Grady D. Too much medicine happens too often: the teachable moment and a call for manuscripts from clinical trainees. JAMA Intern Med. 2014;174(1):8-9. 